\title{
Recognition of Occluded Shapes Using Size Functions
}

\author{
Barbara Di Fabio ${ }^{1}$, Claudia Landi ${ }^{2, \star}$, and Filippo Medri ${ }^{3}$ \\ 1 Dipartimento di Matematica, Università di Bologna, Italy \\ difabio@dm.unibo.it \\ 2 Di.S.M.I., Università di Modena e Reggio Emilia, Italy \\ clandi@unimore.it \\ 3 Dipartimento di Scienze dell'Informazione, Università di Bologna, Italy \\ medri@cs.unibo.it
}

\begin{abstract}
The robustness against occlusions and the ability to perform not only global matching, but also partial matching are investigated in computer vision in order to evaluate the performance of shape descriptors. In this paper we consider the size function shape descriptor, and we illustrate some results about size functions of occluded shapes. Theoretical results indicate that size functions are able to detect a partial matching between shapes by showing a common subset of cornerpoints. Experiments are presented which outline the potential of the proposed approach in recognition tasks in the presence of occlusions.
\end{abstract}

Keywords: Size function, shape occlusion, cornerpoint, Hausdorff distance.

\section{Introduction}

Geometrical-topological methods for shape description and comparison are increasingly studied in computer vision, computer graphics and pattern recognition [1|4|6/7. The common idea underlying the methods of this class is to perform a topological exploration of the shape according to some quantitative geometric properties provided by a real function chosen to extract shape features. For this reason they could be called shape-from-function methods. The attractive feature of shape-from-function techniques is that they concisely capture shape information, in a manner that can be robust to deformation while being able to cope with changes in object viewpoint at a multiresolution level [3]. In this context, size functions have been developed to analyze and compare multidimensional shapes, with application mainly to $2 \mathrm{D}$ digital shapes equipped with scalar functions [8 1821] and 3D digital shapes (represented by surface or volume models) equipped with multivalued functions 2 .

\footnotetext{
* Research partially carried out within the activities of ARCES, University of Bologna, Italy.
} 
Analyzing an image, occlusions can be found every time that foreground objects overlap other objects in the same scene. Therefore, occlusions understanding represents a crucial issue in computer vision.

Although in some contexts, such as stereo matching problems, the presence of occluding patterns may provide useful information (see e.g. [16]), in other settings, such as shape recognition and retrieval problems, the spotlight is on the background partially hidden object and the occlusion is considered to cause a loss of shape features information [19. Therefore, the robustness of shape descriptors against occlusions is a common concern representing a widely researched topic. As far as point based representations of shape are concerned, works on the topic include the partial Hausdorff distance [15] by Huttenlocher et al. to compare portions of point sets, and Wolfson and Rigoutsos' use of geometric hashing 22 applied to point features. For shapes encoded as polylines, the Tanase and Veltkamp's approach [20] is that of computing the dissimilarity between multiple polylines and a polygon using the turning function, while Latecki et al. [17 propose a method based on removing certain parts of a polyline and see whether the objects become more similar without them. As for the region-based shape descriptors, Höynck and Ohm show that using central moments instead of the angular radial transform to extract features improves robustness to occlusions 14. Regarding the shape-from-function methods, Biasotti et al. [5] automatically identify similar sub-parts exploiting a graph-matching technique applied to Reeb graphs.

In this paper we address the partial shape matching problem for size functions. In particular we illustrate the recent results proved in [11] on the behavior of size functions in the presence of occlusions, and their ability to preserve not only global, but also local information. The main result is that an occluded object and a fully visible object share a set of common features in the corresponding size functions. This property can be exploited to support recognition in the presence of occlusions, as shown by the experiments we present here.

The rest of the paper is organized as follows. Section 2 provides a brief overview on size functions. In Section 3 we summarize the relevant theoretical results obtained in [11. Section 4 shows the potential of our approach applied to visible and invisible occlusions. Conclusions and suggestions on future developments end the paper.

\section{Background on Size Functions}

In Size Theory a shape is defined by a pair $(X, \varphi)$, called a size pair, where $X$ is a non-empty compact and locally connected Hausdorff topological space, and $\varphi: X \rightarrow \mathbb{R}$ denotes a continuous function, called a measuring function.

Shapes are studied through their lower level sets $X_{u}=\{p \in X: \varphi(p) \leq u\}, u$ varying in $\mathbb{R}$. Indeed, denoting by $\Delta^{+}$the open half plane $\left\{(u, v) \in \mathbb{R}^{2}: u<v\right\}$, size functions can be defined as follows. 


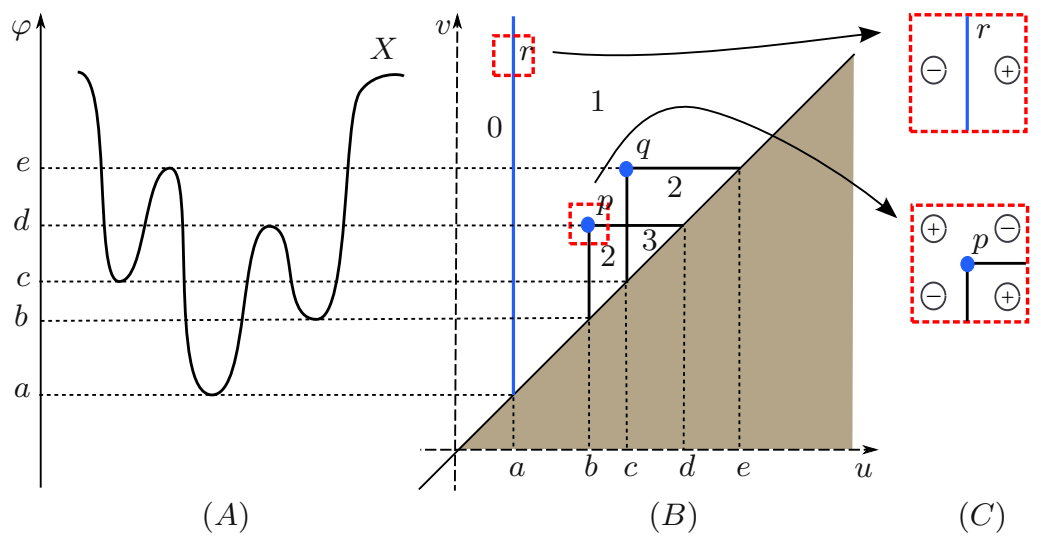

Fig. 1. $(A)$ A size pair $(X, \varphi)$, where $\mathrm{X}$ is the curve represented by a continuous line, and $\varphi: X \rightarrow \mathbb{R}$ is such that $\varphi(P)=y$ for every $P=(x, y) \in X$. (B) The size function associated with $(X, \varphi)$. $(C)$ Computation of multiplicities seen through lens.

Definition 1. The size function associated with the size pair $(X, \varphi)$ is the function $\ell_{(X, \varphi)}: \Delta^{+} \rightarrow \mathbb{N}$ such that, for every $(u, v) \in \Delta^{+}, \ell_{(X, \varphi)}(u, v)$ is equal to the number of connected components in $X_{v}$ containing at least one point of $X_{u}$.

An example of size function is displayed in Fig. 11. In this example we consider the size pair $(X, \varphi)$, where $X$ is the curve of $\mathbb{R}^{2}$ represented by a solid line in Fig. $1(A)$, and $\varphi: X \rightarrow \mathbb{R}$ is the function that associates to each point $P \in X$ its ordinate in the plane. The size function associated with $(X, \varphi)$ is shown in Fig. 1 $(B)$. Here, $\Delta^{+}$, i.e. the domain of the size function, is divided by solid lines, representing the discontinuity points of the size function. These discontinuity points divide $\Delta^{+}$into regions where the size function is constant. The value displayed in each region is the value taken by the size function in that region. For instance, for $c \leq v<d, X_{v}$ has three connected components. Only one of them contains at least one point of $X_{u}$, when $a \leq u<b$; two of them contain at least one point of $X_{u}$, when $b \leq u<c$; all of them contain at least one point of $X_{u}$, when $c \leq u<v<d$. Therefore, when $c \leq v<d, \ell_{(X, \varphi)}(u, v)=1$ for $a \leq u<b ; \ell_{(X, \varphi)}(u, v)=2$ for $b \leq u<c ; \ell_{(X, \varphi)}(u, v)=3$ for $c \leq u<v$.

An equivalent representation of size function is given by countable collections of cornerpoints (proper and at infinity), with multiplicity 13. A proper cornerpoint for $\ell_{(X, \varphi)}$ is a point $(u, v) \in \Delta^{+}$encoding the level $u$ at which a new connected component is born and the level $v$ at which it get merged to another connected component. A cornerpoint at infinity just encodes the level $u$ at which a new connected component of $X$ is born. Formally they can be defined as follows.

Definition 2. We call a proper cornerpoint for $\ell_{(X, \varphi)}$ any point $p=(u, v) \in$ $\Delta^{+}$such that the number $\mu_{X}(p)=\lim _{\epsilon \rightarrow 0^{+}} \mu_{X}^{\epsilon}(p)$, with $\mu_{X}^{\epsilon}(p)$ equal to $\ell_{(X, \varphi)}(u+\epsilon, v-\epsilon)-\ell_{(X, \varphi)}(u-\epsilon, v-\epsilon)-\ell_{(X, \varphi)}(u+\epsilon, v+\epsilon)+\ell_{(X, \varphi)}(u-\epsilon, v+\epsilon)$, 
is strictly positive. Moreover, we call a cornerpoint at infinity for $\ell_{(X, \varphi)}$ any vertical line $r$, with equation $u=k$, such that the number $\mu_{X}(r)=\lim _{\epsilon \rightarrow 0^{+}} \mu_{X}^{\epsilon}(r)$, with $\mu_{X}^{\epsilon}(r)$ equal to

$$
\ell_{(X, \varphi)}(k+\epsilon, 1 / \epsilon)-\ell_{(X, \varphi)}(k-\epsilon, 1 / \epsilon),
$$

is strictly positive.

Fig. $1(C)$ zooms in on some cornerpoints to explain how their multiplicity is computed. For instance, the alternating sum of the size function values at four points around $p$ is $2-1-1+1$, giving $\mu_{X}(p)=1$. The alternating sum of the size function values at two points next to $r$ is $1-0$, giving $\mu_{X}(r)=1$.

Cornerpoints allow us to translate the comparison between size functions into distances between sets of points such as the Hausdorff metric or the matching distance, in a way that is robust against deformations 1013 .

From the computational point of view, the existing algorithm for computing size functions 9] requires the construction of a size graph $(G, \varphi)$, where $G=$ $(V(G), E(G))$ is a finite graph, with $V(G)$ and $E(G)$ the set of vertices and edges respectively, and $\varphi: V(G) \rightarrow \mathbb{R}$ is a measuring function labeling the nodes of the graph. In this paper we deal with binary 2D images, so $V(G)$ corresponds to image pixels and $E(G)$ represents the 8-neighborhood connectivity.

Once the size graph has been built, the computational complexity for computing the set of cornerpoints is $O(n \log n+m \cdot \alpha(2 m+n, n))$, where $n$ and $m$ are the number of vertices and edges in the size graph, respectively, and $\alpha$ is the inverse of the Ackermann function.

Finally, in order to compare two size functions, encoded as collections of cornerpoints, each of cardinality $N$, the complexity of a simple implementation of the Hausdorff distance is $O\left(N^{2}\right)$, but a more efficient algorithm can reduce time complexity to $O(N \log N)$. We remark that, in general, $N$ is always very much smaller than $n$ and $m$.

\section{Size Functions and Occluded Shapes}

We model the presence of occlusions in a shape as follows. The object of interest $A$ is occluded by an object $B$, so that $X=A \cup B$ is the object visible after occlusion. These sets are assumed to be locally connected compact Hausdorff spaces.

The shapes of $X, A$, and $B$ are analyzed through the size functions $\ell_{(X, \varphi)}$, $\ell_{\left(A, \varphi_{\mid A}\right)}$, and $\ell_{\left(B, \varphi_{\mid B}\right)}$, respectively, where $\varphi: X \rightarrow \mathbb{R}$ is the continuous function chosen to extract the shape features.

The main theorem in [11] establishes a necessary and sufficient algebraic condition such that a (Mayer-Vietoris) relation exists among the size functions associated with $X, A, B$, and $A \cap B$. To be more precise, denoting by $\check{H}$ the Čech homology with coefficients in a field $K$, and by $\alpha_{v}: \check{H}_{0}\left((A \cap B)_{v}\right) \rightarrow \check{H}_{0}\left(A_{v}\right) \oplus$ $\check{H}_{0}\left(B_{v}\right), \alpha_{v, u}: \check{H}_{0}\left((A \cap B)_{v},(A \cap B)_{u}\right) \rightarrow \check{H}_{0}\left(A_{v}, A_{u}\right) \oplus \check{H}_{0}\left(B_{v}, B_{u}\right)$ the homomorphisms belonging to the Mayer-Vietoris sequence of the triad $\left(X_{v}, A_{v}, B_{v}\right)$ and to 
the relative Mayer-Vietoris sequence of the triad $\left(\left(X_{v}, X_{u}\right),\left(A_{v}, A_{u}\right),\left(B_{v}, B_{u}\right)\right)$, respectively, the following statement can be proved.

Theorem 1. For every $(u, v) \in \Delta^{+}$, it holds that

$$
\ell_{(X, \varphi)}(u, v)=\ell_{\left(A, \varphi_{\mid A}\right)}(u, v)+\ell_{\left(B, \varphi_{\mid B}\right)}(u, v)-\ell_{\left(A \cap B, \varphi_{\mid A \cap B}\right)}(u, v)
$$

if and only if $\operatorname{dim} \operatorname{ker} \alpha_{v}=\operatorname{dim} \operatorname{ker} \alpha_{v, u}$.

The relation among the size functions $\ell_{(X, \varphi)}, \ell_{\left(A,\left.\varphi\right|_{A}\right)}, \ell_{\left(B,\left.\varphi\right|_{B}\right)}$ and $\ell_{\left(A \cap B,\left.\varphi\right|_{A \cap B}\right)}$, stated in Theorem 1, can be translated into a relation among their cornerpoints. More precisely, the coordinates of cornerpoints for $\ell_{(X, \varphi)}$ are always coordinates of cornerpoints for $\ell_{\left(A,\left.\varphi\right|_{A}\right)}$ or $\ell_{\left(B,\left.\varphi\right|_{B}\right)}$ or $\ell_{\left(A \cap B,\left.\varphi\right|_{A \cap B}\right)}([11$, Thm. 5.4-5.5). Moreover, under proper assumptions, cornerpoints for $\ell_{(X, \varphi)}$ are cornerpoints for $\ell_{\left(A,\left.\varphi\right|_{A}\right)}$ or $\ell_{\left(B,\left.\varphi\right|_{B}\right)}$ or both (11], Cor. 5.2). Fig. 2] illustrates these results on an example for which relation (11) holds everywhere in $\Delta^{+}$.
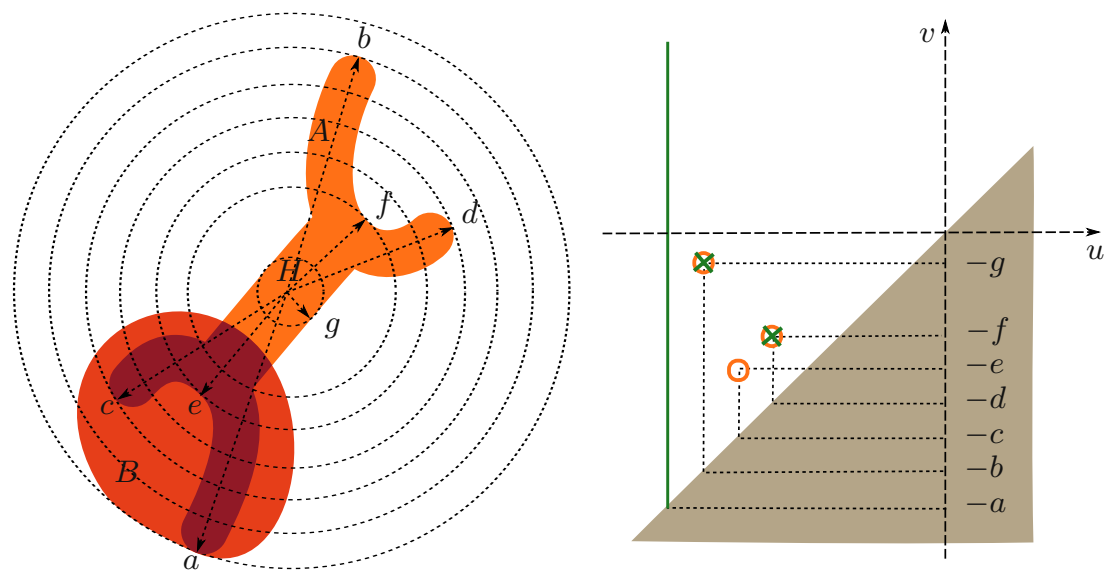

Fig. 2. Left: A "bone" shaped object $A$ is occluded by another object $B$. The resulting occluded object $X=A \cup B$ is analyzed taking $\varphi: X \rightarrow \mathbb{R}, \varphi(P)=-\|P-H\|$. Right: The only cornerpoint at infinity for $\ell_{(X, \varphi)}, u=-a$, is also a cornerpoint at infinity for both $\ell_{\left(A,\left.\varphi\right|_{A}\right)}$ and $\ell_{\left(B,\left.\varphi\right|_{B}\right)}$; proper cornerpoints for $\ell_{(X, \varphi)}(\times)$ belong to the set of cornerpoints for $\ell_{\left(A,\left.\varphi\right|_{A}\right)}(\bigcirc)$.

\section{Experimental Results}

In this section we are going to describe the results we have achieved in some preliminary experiments. In order to analyze the potential of our approach in the recognition of occluded shapes, we have considered both visible and invisible occlusions. To perform our tests we have worked with filled images from the MPEG-7 dataset 23. In all the experiments, the occluding pattern is a rectangular shape occluding from the top, or the left, by an area we increasingly vary 
from $10 \%$ to $60 \%$ of the height or width of the bounding box of the original shape. For both the original shapes and the occluded ones, size functions are always computed with respect to a family of eight measuring functions having only the set of black pixels as domain. They are defined as follows: four of them as the distance from the line passing through the origin (top left point of the bounding box), rotated by an angle of $0, \frac{\pi}{4}, \frac{\pi}{2}$ and $\frac{3 \pi}{4}$ radians, respectively, with respect to the horizontal position; the other four as minus the distance from the same lines, respectively.

In the case of visible occlusions, with reference to the notation used in our theoretical setting, we are considering $A$ as the original shape, $B$ as a black rectangle, and $X$ as the occluded shape generated by their union.

In Table 1 (column 3) a "device1" shape is depicted with one of its eight size functions. By comparison with the size function of the same object occluded from the top (column 1), or from the left (column 2), with respect to the same measuring function, it is easily seen that they present common substructures, since some cornerpoints are preserved after occlusions.

In Table 2, for different levels of occlusion, each 3D bar chart displays, along the z-axis, the percentage of common cornerpoints between the set of size functions associated with 70 shapes, each chosen from a different class of the MPEG-7 dataset (x-axis), and the set of size functions associated with the 70 occluded ones (y-axis). Note that, for each occluded shape, the highest bar is always on the diagonal, that is, where the occluded object is compared with the corresponding original one.

It has to be expected that, when a shape is not only occluded but also deformed, it will not be possible to find a common subset of cornerpoints between the original shape and the occluded one, since the deformation has slightly changed the cornerpoints position (see examples in Table 1, columns 3-5). To test the behavior of size functions when both occlusions and deformations are

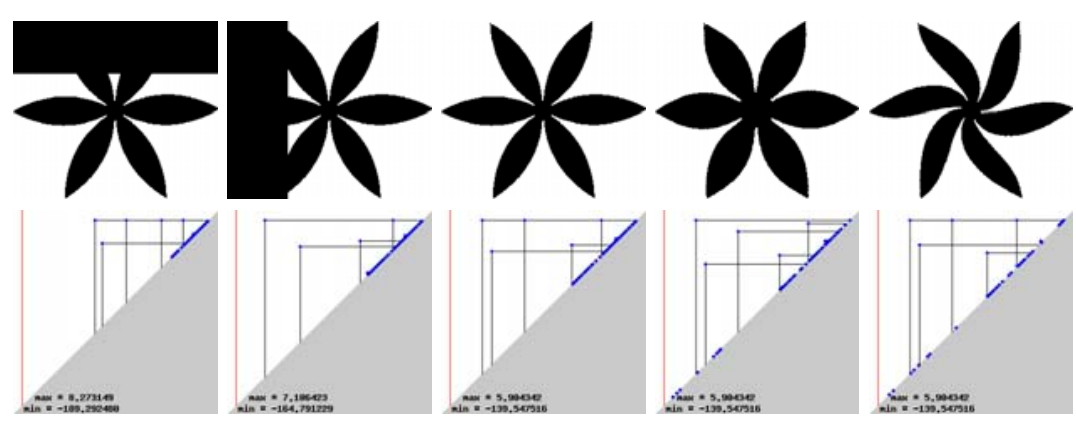

Table 1. Row 1: in columns 3-5, three "device1" shapes; in columns 1-2, the same "device1" shape as depicted in column 3, occluded from the top and from the left, respectively. Row 2: corresponding size functions associated with the same measuring function. 

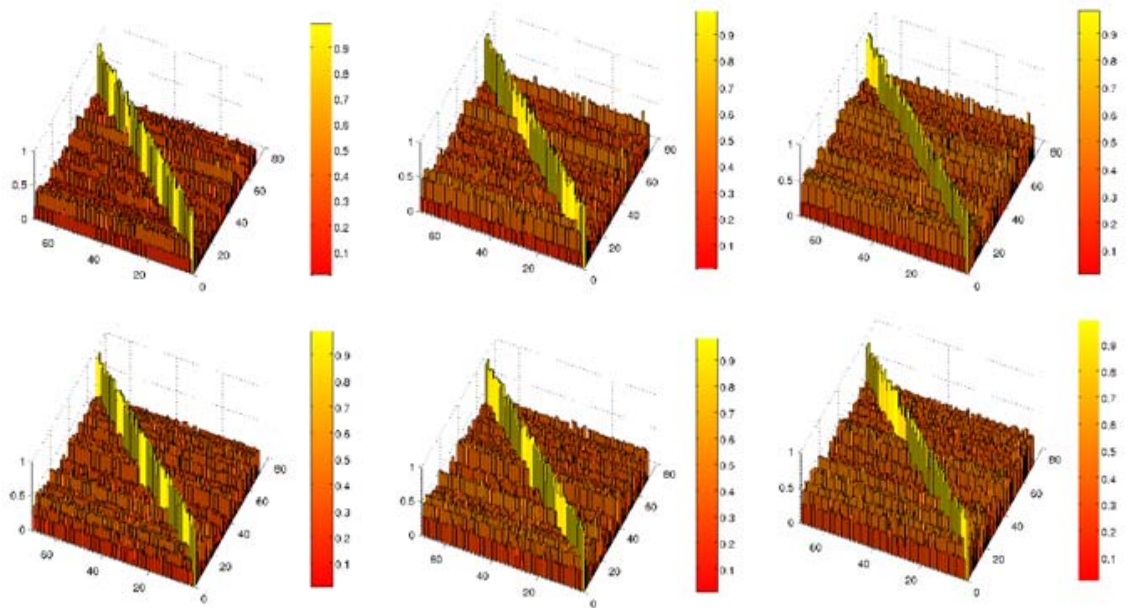

Table 2. 3D bar charts displaying, in the case of visible occlusions, the percentage of common cornerpoints (z-axis) between 70 occluded shapes (y-axis) and the 70 original ones (x-axis) correspondingly ordered. Row 1: Shapes are occluded from top by $20 \%$ (column 1), by $40 \%$ (column 2), by $60 \%$ (column 3). Row 2: Shapes are occluded from the left by $20 \%$ (column 1), by $40 \%$ (column 2 ), by $60 \%$ (column 3 ).

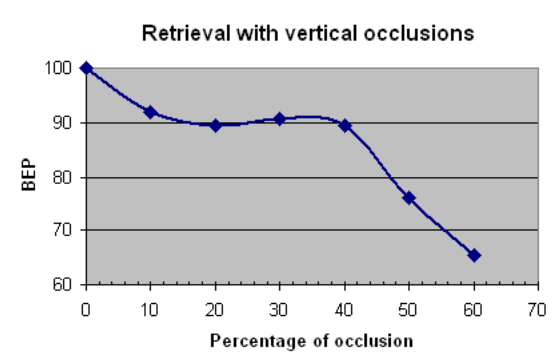

(a)

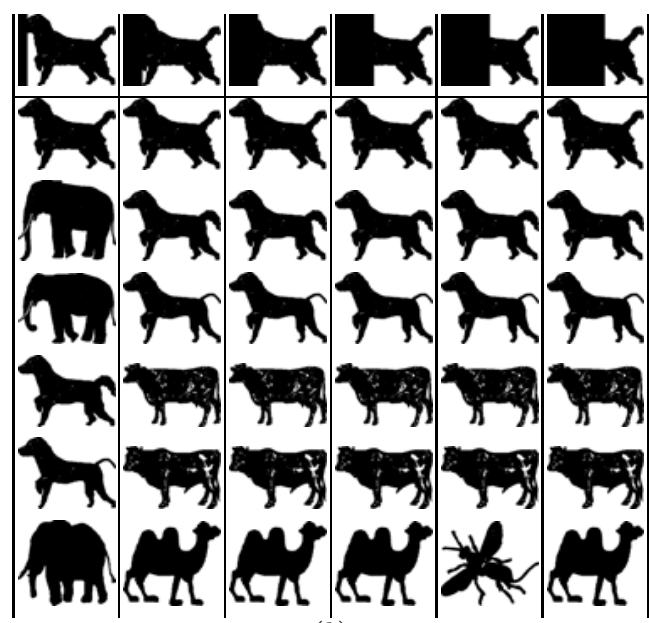

(b)

Table 3. (a) The variation of retrieval performance when the occlusion area increases from the left; (b) Top retrieval results for a "dog" shape partially occluded from the left. Results are depicted in every column in increasing order of distance from the query.

introduced, we perform a retrieval test with the training set consisting of 75 images: three instances chosen from 25 different classes. The test set contains 25 occluded images, each taken from a different class. Each of them is taken 

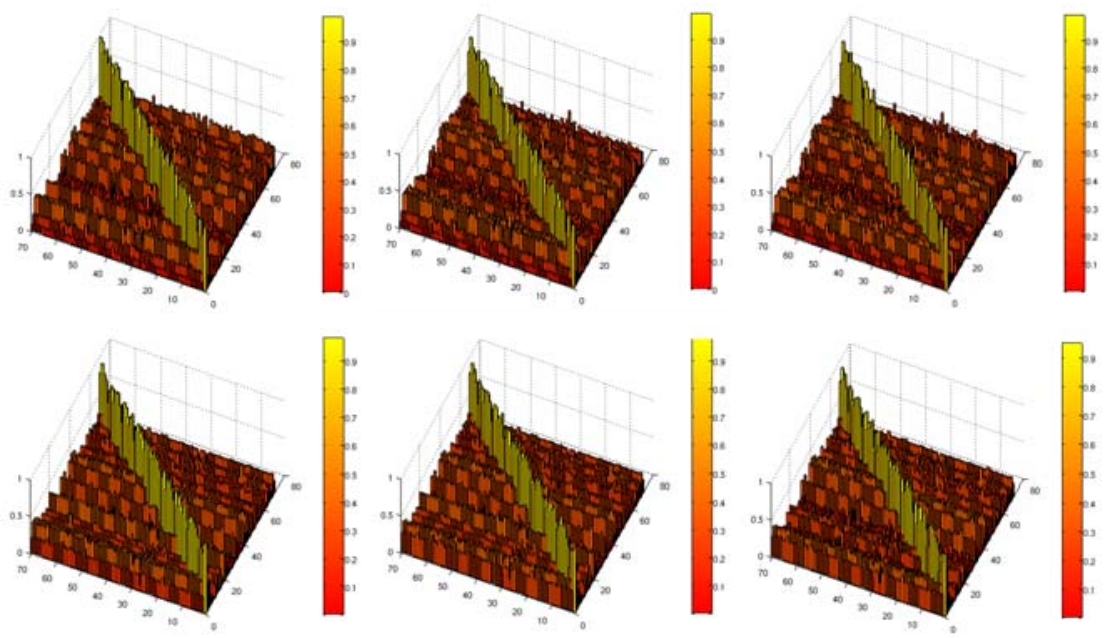

Table 4. 3D bar charts displaying, in the case of invisible occlusions, the percentage of common cornerpoints (z-axis) between 70 occluded shapes (y-axis) and the 70 original ones (x-axis) correspondingly ordered. Row 1: Shapes are occluded from top by $20 \%$ (column 1), by $30 \%$ (column 2), by $40 \%$ (column 3). Row 2: Shapes are occluded from the left by $20 \%$ (column 1 ), by $30 \%$ (column 2 ), by $40 \%$ (column 3 ).

as a query and is matched with all the images in the training set. Comparison is performed by calculating the sum of the eight Hausdorff distances between the sets of cornerpoints for the size functions associated with the corresponding eight measuring functions. The retrieval is evaluated using the Bull's Eye Performance (BEP) criterion. The BEP is measured by the correct retrievals among the top $2 N$ retrievals, where $N$ is the number of relevant (or similar) shapes to the query in the database. The effect of an increasing occlusion by a vertical rectangle on the retrieval performance is described by the graphs in Table $3(a)$, while examples of query tests are exhibited in Table $3(b)$.

When invisible occluding patterns are considered, with reference to the notation used in our theoretical setting, we take $X$ as the original shape, $A$ as the the occluded shape, and $B$ as the invisible part of $X$. In this case, a comparison between cornerpoints of size functions, analogous to that of Table 2 is given in Table 4. The percentages of occlusion, from the top (row 1) and from the left (row 2), here vary from 20 to 40 (columns 1-3). Graphs in Table 5 (a) illustrate the recognition trend when, increasing the percentage of occlusion, we compare two shapes through the Hausdorff distance between their size functions, under the following convention. When the original shape is disconnected by the occlusion, we retain only the connected component of greatest area. This choice allows us to obtain always a finite Hausdorff distance, but determines a high loss of shape information even when the percentage of occlusion is low (see examples in Table $5(b))$. 
Rate of recognition with horizontal occlusions

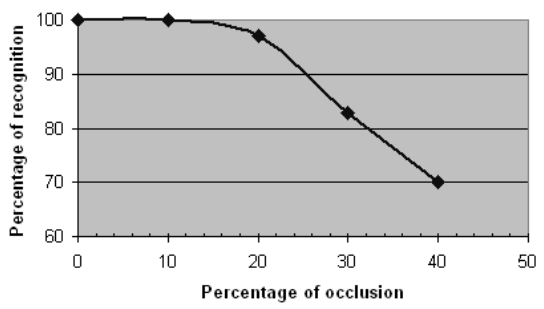

Rate of recognition with vertical occlusions

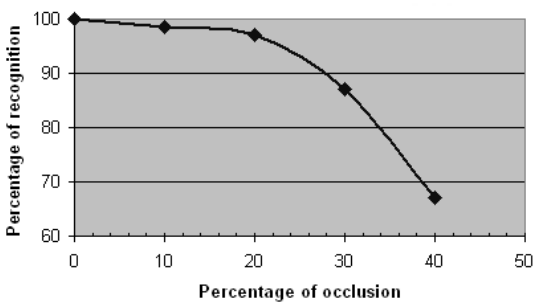

(a)

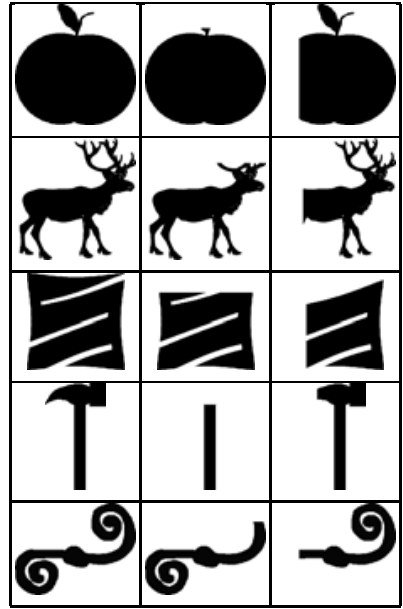

(b)

Table 5. (a) Recognition trend when the occluded area from the top and from the left increases. (b) The first column: some instances from the MPEG-7 dataset. The second and third columns: by $20 \%$ occluded from the top and from the left, respectively.

\section{Discussion}

In this paper we have proposed a method to assess partial matching using size functions. As demonstrated, recognition in the presence of occlusions amounts to detect common subsets of cornerpoints in size functions. The experimental results show that this method is effective both with visible and invisible occlusions. However, when deformations are added to occlusions, the Hausdorff distance between size functions seems not to be robust enough for recognition or retrieval tasks. The reason is that it works globally on the whole set of cornerpoints and therefore it is not able to detect substructures. As a consequence, an important open question is how to automatically detect similar substructures in size functions when cornerpoints can be distorted. This question could be addressed in a future research combining the results shown in this paper with the polynomial representation of size functions 12 .

\section{References}

1. Biasotti, S., Cerri, A., Frosini, P., Giorgi, D., Landi, C.: Multidimensional Size Functions for Shape Comparison. J. Math. Imaging Vis. 32(2), 161-179 (2008)

2. Biasotti, S., Cerri, A., Giorgi, D.: $k$-dimensional size functions for shape description and comparison. In: Proc. of ICIAP 2007, pp. 345-352 (2007) 
3. Biasotti, S., De Floriani, L., Falcidieno, B., Frosini, P., Giorgi, D., Landi, C., Papaleo, L., Spagnuolo, M.: Describing shapes by geometrical-topological properties of real functions. ACM Computing Surveys 40(4), 12:1-12:87 (2008)

4. Biasotti, S., Giorgi, D., Spagnuolo, M., Falcidieno, B.: Reeb graphs for shape analysis and applications. Theor. Comput. Sci. 392(1-3), 5-22 (2008)

5. Biasotti, S., Marini, S., Spagnuolo, M., Falcidieno, B.: Sub-part correspondence by structural descriptors of 3D shapes. Computer-Aided Design 38(9), 1002-1019 (2006)

6. Carlsson, G., Zomorodian, A., Collins, A., Guibas, L.: Persistence barcodes for shapes. International Journal of Shape Modeling 11, 149-187 (2005)

7. Cazals, F., Chazal, F., Lewiner, T.: Molecular shape analysis based upon the MorseSmale complex and the Connolly function. In: Proc. of SCG 2003, pp. 351-360 (2003)

8. Cerri, A., Ferri, M., Giorgi, D.: Retrieval of trademarks images by means of size functions. Graphical Models 68, 451-471 (2006)

9. d'Amico, M.: A New Optimal Algorithm for Computing Size Functions of Shapes. In: Proc. of CVPRIP Algoritms III, pp. 107-110 (2000)

10. d'Amico, M., Frosini, P., Landi, C.: Natural pseudo-distance and optimal matching between reduced size functions. Acta Appl. Math. (digitally available)

11. Di Fabio, B., Landi, C.: Čech homology for shape recognition in the presence of occlusions (preprint), http://arxiv.org/PS_cache/arxiv/pdf/0807/0807.0796v1.pdf

12. Ferri, M., Landi, C.: Representing size functions by complex polynomials. Proc. Math. Met. in Pattern Recognition 9, 16-19 (1999)

13. Landi, C., Frosini, P.: New pseudodistances for the size function space. In: Proc. SPIE, Vision Geometry VI, vol. 3168, pp. 52-60 (1997)

14. Höynck, M., Ohm, J.R.: Shape retrieval with robustness against partial occlusion. In: Proc. of IEEE ICASSP 2003, vol. 3, pp. 593-596 (2003)

15. Huttenlocher, D.P., Klanderman, G.A., Rucklidge, W.J.: Comparing images using the Hausdorff distance. IEEE Trans. PAMI 15, 850-863 (1993)

16. Intille, S.S., Bobick, A.F.: Disparity-space images and large occlusion stereo. In: Proc. of ECCV 1994, vol. II, pp. 179-186 (1994)

17. Latecki, L.J., Lakämper, R., Wolter, D.: Optimal partial shape similarity. Image Vision Comput. 23(2), 227-236 (2005)

18. Stanganelli, I., Brucale, A., Calori, L., Gori, R., Lovato, A., Magi, S., Kopf, B., Bacchilega, R., Rapisarda, V., Testori, A., Ascierto, P.A., Simeone, E., Ferri, M.: Computer-aided diagnosis of melanocytic lesions. Anticancer Res. 25, 4577-4582 (2005)

19. Veltkamp, R.C.: Shape matching: similarity measures and algorithms. In: Proc. of Shape Modeling International 2001, pp. 188-197 (2001)

20. Tanase, M., Veltkamp, R.C.: Part-based shape retrieval. In: Proc. of MULTIMEDIA 2005, pp. 543-546 (2005)

21. Uras, C., Verri, A.: Computing size functions from edge maps. Internat. J. Comput. Vis. 23(2), 169-183 (1997)

22. Wolfson, H.J., Rigoutsos, I.: Geometric Hashing: An Overview. IEEE Computational Science and Engineering 4(4), 10-21 (1997)

23. http://www.imageprocessingplace.com/root_files_V3/image_databases.htm 